\title{
GEOMETRIC AND SPECTRAL ESTIMATES BASED ON SPECTRAL RICCI CURVATURE ASSUMPTIONS
}

\author{
GILLES CARRON AND CHRISTIAN ROSE
}

\begin{abstract}
We obtain a Bonnet-Myers theorem under a spectral condition: a closed Riemannian $\left(M^{n}, g\right)$ manifold for which the lowest eigenvalue of the Ricci tensor $\rho$ is such that the Schrödinger operator $(n-2) \Delta+\rho$ is positive has finite fundamental group. Further, as a continuation of our earlier results, we obtain isoperimetric inequalities from Kato-type conditions on the Ricci curvature. We also obtain the Kato condition for the Ricci curvature under purely geometric assumptions.
\end{abstract}

\section{INTRODUCTION}

1.1. Several classical results in differential geometry state geometric, spectral or topological estimates for a closed Riemannian manifold assuming pointwise curvature constraints. Prominent examples of such results depending on some lower bound on the Ricci curvature are the theorems of Bonnet-Myers, Bochner, estimates of isoperimetric constants, and eigenvalue estimates for the LaplaceBeltrami operator, as well as bounds on the Betti numbers. An intriguing question is whether the assumption of a sharp lower Ricci curvature bound can be relaxed to variable curvature bounds instead. Of course, one can ask why this should be interesting at all: the Ricci tensor as a continuous function on a compact set is bounded below anyway. The answer lies in the quantitative nature of our question: if we disturb the curvature assumptions of the classical theorems by a small portion of "bad" curvature, how do the classical estimates change? Within this context, the treatment of families of manifolds are of importance: One can ask for uniform properties of all of the members of the family. Indeed, one could make assumptions on the infimum of the Ricci curvatures of all the members and derive estimates, but they will be not stable under small changes and much weaker than perturbation statements. Results in this direction have been obtained by K-D. Elworthy and S. Rosenberg, [15]. From the pioneering work of S. Gallot, there have been a lot of results based on integral pinching conditions for the Ricci curvature $([18,17,2,25,26,28,27,14,29,35,38,3,4])$.

Recently, we have obtained heat kernel and eigenvalue estimates assuming only a Kato condition on the negative part of Ricci curvature [31, 11]. It has been shown

Date: August 22, 2018.

1991 Mathematics Subject Classification. Primary 53C21, 58J35, secondary: 58C40, 58J50 .

${ }^{0}$ Mots clés: Inégalité de Sobolev, inégalités isopérimètriques,

${ }^{0}$ Key words: Sobolev inequality, isoperimetric inequality. 
that this type of condition is somewhat weaker that the integral pinching assumption made by S. Gallot.

1.2. Let's us introduce several notations and conventions: In all that follows $\left(M^{n}, g\right)$ is always a closed Riemannian manifold of dimension $n \in \mathbb{N}$, i.e., compact and without boundary, and vol, respectively $\mathcal{A}$, denote the $n$-, respectively $(n-1)$-dimensional Hausdorff measure on $M$. Denote by Ric the Ricci tensor of $M$ considered pointwise as an symmetric endomorphism of $T^{*} M$. We let

$$
\rho: M \rightarrow \mathbb{R}, x \mapsto \min \left\{\sigma\left(\operatorname{Ric}_{x}\right)\right\},
$$

where $\sigma(A)$ denotes the spectrum of an operator $A$. We have

$$
\operatorname{Ric}_{x} \geq \rho(x) \operatorname{Id}_{T_{x} M}
$$

or $\mathrm{Ric}_{x} \geq \rho(x) g_{x}$ in the sense of quadratic forms on the tangent space at $x \in M$. If $x$ is a real number, its negative part will be denoted by $x_{-}=\max \{0,-x\}$, for instance:

$$
\operatorname{Ric}_{x} \geq-\rho_{-}(x) g_{x} \text { or } \operatorname{Ric}_{x} \geq \lambda g-(\rho(x)-\lambda)_{-} g_{x} .
$$

As usual, we denote by $\Delta \geq 0$ the non-negative Laplace-Beltrami operator acting on functions defined on $M$. This operator has purely discrete spectrum

$$
\sigma(\Delta)=\left\{\lambda_{i} \mid i \in \mathbb{N}_{0}\right\},
$$

where

$$
0=\lambda_{0}<\lambda_{1} \leq \lambda_{2} \leq \ldots
$$

1.3. Our first result is based on an a spectral assumption for a Schrödinger operator:

Theorem A. Let $\left(M^{n}, g\right)$ be a closed Riemannian manifold of dimension $n \geq 3$.

i) For any $\epsilon \in\left[0, \frac{3}{n+4}\right)$, there is an explicit constant $C(n, \epsilon)>0$ satisfying $\lim _{\epsilon \rightarrow 0+} C(n,, \epsilon)=1$, such that if the Schrödinger operator

$$
\epsilon \Delta+\rho-(n-1) k^{2}
$$

is non negative, then we have $\operatorname{diam}(M, g) \leq C(n, \epsilon) \frac{\pi}{k}$.

ii) If for some $\epsilon \in\left[0, \frac{1}{n-2}\right]$, the bottom of the spectrum of Schrödinger operator

$$
\epsilon \Delta+\rho
$$

is positive, then the fundamental group of $M$ is finite.

Remarks 1.1. (1) This result is a weak generalization of the Bonnet-Myers theorem that states that if a complete Riemannian manifold $\left(M^{n}, g\right)$ satisfies

$$
\operatorname{Ric} \geq(n-1) k^{2},
$$

then $M$ is closed, $\operatorname{diam}(M, g) \leq \pi$ and the fundamental group of $M$ is finite. 
(2) With respect to integral bound on curvature, the sharp result has been obtained by E. Aubry ([2]): Let $2 p>n \geq 3$, there is an $\epsilon(p, n)>0$ such that if a complete Riemannian manifold $\left(M^{n}, g\right)$ satisfies

$$
\frac{1}{\operatorname{vol}(M)} \int_{M}(\rho-(n-1))_{-}^{p} \mathrm{dvol} \leq \epsilon(p, n)
$$

then $M$ is closed, there is an explicit estimate for the diameter of $(M, g)$ and the fundamental group of $M$ is finite.

(3) The first result is obtained in two stages. One first proves a Sobolev inequality (using the idea of D. Bakry and I. Mondello ([6, 23])), and then uses a result of D. Bakry and M. Ledoux ([5]).

(4) For $n=3$, Bour and Carron ([8]) have shown that if the operator

$$
2 \Delta+\rho
$$

is positive, then the universal cover of $M^{3}$ is $\mathbb{S}^{3}$.

(5) We do not know wether the threshold $1 /(n-2)$ is sharp, but we believe it is not.

(6) We do not know wether a complete Riemannian manifold $\left(M^{n}, g\right)$ such that for some positive $\epsilon<1 /(n-2)$, the Schrödinger operator

$$
\epsilon \Delta+\rho
$$

is positive must be closed. However, the proof of the theorem would show that if a complete Riemannian manifold $\left(M^{n}, g\right)$ of dimension $n \geq 3$ satisfies a uniform lower bound on the Ricci curvature of the form $\operatorname{Ric}_{x} \geq$ $-(n-1) k^{2}$, a non collapsing assumption $\inf _{x} \operatorname{vol} B(x, 1)>0$, and the fact that for some $\epsilon \in\left[0, \frac{1}{n-2}\right)$, the bottom of the spectrum of Schrödinger operator $\epsilon \Delta+\rho$ is positive, then $M$ is closed.

The spectral assumption made in the above theorem are classically implied by a Kato condition on the Ricci curvature. Let $\left(P_{t}=e^{-t \Delta}\right)_{\{t \geq 0\}}$ be the heat semigroup and $H(t, x, y)$ the heat kernel, that is, for $f \in L^{1}(M)$ and $t>0$, one has

$$
x \in M:\left(P_{t} f\right)(x)=\left(e^{-t \Delta} f\right)(x)=\int_{M} H(t, x, y) f(y) \operatorname{dvol}(y) .
$$

For a measurable $V: M \rightarrow[0, \infty]$ and $T>0$, the Kato constant of $V$ is given by

$$
\kappa_{T}(V):=\sup _{\ell \in \mathbb{N}}\left\|\int_{0}^{T} P_{t}(V \wedge \ell) \mathrm{d} t\right\|_{\infty},
$$

(Note that the semigroup maps $L^{\infty}(M)$ to itself, such that the truncation procedure ensures that the quantity is well-defined.) where $a \wedge b$ denotes the minimum of the numbers $a, b$. We say here that a measurable $V \geq 0$ satisfies the Kato condition if there is a $T>0$ such that

$$
\kappa_{T}(V)<1
$$

The Kato condition is very powerful in proving, e.g., semiboundedness of the operator $\Delta-V$, and mapping properties of the corresponding semigroups. E.g., if 
the heat semigroup is ultracontractive, the perturbed semigroup will be, too.

Remarks 1.2. There is another constant used in the literature also known as the Kato constant: for a measurable, non-negative $V: M \rightarrow[0, \infty]$, for some $L>0$ we let

$$
c_{L}(V):=\sup _{n \in \mathbb{N}}\left\|(\Delta+L)^{-1}(V \wedge n)\right\|_{\infty} .
$$

It is not hard to prove that $c_{L}$ and $\kappa_{T}$ are related by the formula [20]

$$
\left(1-\mathrm{e}^{-L T}\right) c_{L}(V) \leq \kappa_{T}(V) \leq \mathrm{e}^{L T} c_{L}(V),
$$

meaning that the behavior of $\kappa_{T}(V)$ for $T \rightarrow 0$ controls the behavior of $c_{L}(V)$ for $L \rightarrow \infty$ and vice versa.

Remarks 1.3. (1) The Kato class was introduced in [21] and further investigated in [1, 34], originally for the Laplacian in $\mathbb{R}^{n}$. There, a function $V$ is in the Kato class if $c_{T}(V) \rightarrow 0$ as $T \rightarrow \infty$. Actually, the condition was phrased in terms of truncated heat kernels, see [37].

(2) The Kato condition has been extended to a certain class of measure perturbations of a general regular Dirichlet form in [36]. In this article, the Kato class consists of those measures $\mu$ for which $c_{T}(\mu)<1$ for large $T$. In particular, it was shown that in this context mapping properties of the semigroups carry over from the unperturbed to the perturbed. For instance, if for some $\mu>0$, one has $\kappa_{T}(V) \leq\left(1-e^{-\mu T}\right)$, then

$$
\left\|e^{-T(\Delta-V)}\right\|_{L^{1} \rightarrow L^{1}} \leq e^{\mu T} .
$$

In particular, the bottom of the spectrum of the Schrödinger operator $\Delta-V$ is bounded from below by $-\mu$.

Corollary B. If $\left(M^{n}, g\right)$ is a closed Riemannian manifold of dimension $n \geq 3$ such that for some $\lambda>0$ and $T>0$ one has

$$
\kappa_{T}\left((\rho-\lambda)_{-}\right)<\frac{1-e^{-(n-2) \lambda T}}{n-2},
$$

then the fundamental group of $M$ is finite.

One can also obtain a Lichnerowicz-type estimate for the first non-zero eigenvalue. One of our results is the following:

Proposition C. Let $\left(M^{n}, g\right)$ be a closed manifold of dimension $n \geq 3$ such that for some $0 \leq k<1$, we have

$$
\kappa_{T}\left((\rho-(n-1))_{-}\right) \leq \frac{n}{n-1}\left(1-e^{-T \frac{(n-1)^{2}}{n}\left(1-k^{2}\right)}\right) .
$$

Then, the first eigenvalue $\lambda_{1}(M)$ satisfies

$$
\lambda_{1}(M) \geq n k^{2} .
$$


In [11], it has been asked wether a control of the Ricci curvature in some Kato class yields some isoperimetric inequality. The second main result of this paper provided such a result using an idea of M. Ledoux ([22]). The first result is a reverse Cheeger inequality in the spirit of P. Buser ([9]). Recall that the Cheeger constant of $\left(M^{n}, g\right)$ is defined by:

$$
\mathrm{h}(M, g):=\inf _{\Omega} \frac{\mathcal{A}(\partial \Omega)}{\operatorname{vol}(\Omega)}
$$

where the infimum is taken over all $\Omega \subset M$ having smooth boundary $\partial \Omega$ and $\operatorname{vol}(\Omega) \leq \operatorname{vol}(M) / 2$. J. Cheeger $([12])$ has shown that one always has

$$
\frac{\mathrm{h}^{2}(M, g)}{4} \leq \lambda_{1}
$$

and P. Buser proved that if the Ricci curvarture satisfies

$$
\operatorname{Ric}_{x} \geq-(n-1) k^{2}
$$

then one has

$$
\lambda_{1} \leq c(n)\left(k \mathrm{~h}(M, g)+\mathrm{h}^{2}(M, g)\right) .
$$

Proposition D. Let $\left(M^{n}, g\right)$ be a closed Riemannian manifold of dimension $n \geq 2$ such that for some $T>0$, we have

$$
\kappa_{T}\left(\rho_{-}\right) \leq \frac{1}{16 n} .
$$

There is a explicit constant $c_{n}$ such that

$$
\lambda_{1}(M) \leq c_{n}\left(\frac{1}{\sqrt{T}} \mathrm{~h}(M)+\mathrm{h}^{2}(M)\right) .
$$

Using the eigenvalue estimates from [11], the estimate above yields lower bounds for the Cheeger constants under Kato-type assumptions on the negative part of the Ricci curvature. Note that assuming a lower bound $\operatorname{Ric}_{x} \geq-(n-1) k^{2}$ on the Ricci curvature yields $\kappa_{T}\left(\rho_{-}\right) \leq T(n-1) k^{2}$, hence our result is a slight generalization of Buser's one. Furthermore, we obtain isoperimetric inequalities. Recall that for $p \in[1, \infty)$, the $p$-isoperimetric constant of $M$ is defined by

$$
I_{p}(M):=\inf _{\Omega} \frac{\mathcal{A}(\partial \Omega)}{\operatorname{vol}(\Omega)^{1-1 / p}},
$$

where the infimum is taken over all $\Omega \subset M$ having smooth boundary $\partial \Omega$ and $\operatorname{vol}(\Omega) \leq \operatorname{vol}(M) / 2$.

Theorem E. Let $\left(M^{n}, g\right)$ be a closed Riemannian manifold of dimension $n \geq 2$ and diameter at most $D>0$. Assume that for some $p>1$, there is an $\mathbf{I}>0$ such that

$$
D^{2 p-2} \kappa_{T}\left(\rho_{-}^{p}\right) \leq \mathbf{I}^{p}
$$

and let $\xi=\max \left\{\frac{D}{\sqrt{T}},(16 n \mathbf{I})^{\frac{p}{2 p-2}}\right\}$. Then we have

$$
D \operatorname{vol}(M)^{1 / n} c_{n}^{1+\xi} I_{n}(M) \geq 1 .
$$


Our last result is that the assumptions made on the Kato constant of the Ricci curvature are implied under a control that depends on the volume of geodesic balls rather than the heat kernel.

Theorem F. There is a positive constant $\eta_{n}$ such that when $\left(M^{n}, g\right)$ is a closed Riemannian manifold of diameter $D$ for which all $x \in M$ satisfy:

$$
\int_{0}^{D} r\left(\frac{1}{\operatorname{vol} B(x, r)} \int_{B(x, r)} \rho_{-}\right) d r \leq \epsilon_{n}
$$

then $\kappa_{D^{2}}\left(\rho_{-}\right) \leq \frac{1}{16 n}$ and the first Betti number of $M$ is smaller then $n$ :

$$
b_{1}(M) \leq n \text {. }
$$

This paper is organized as follows. Sections 2.1 and 2.2 are devoted to present a Sobolev inequality under a spectral hypothesis on compact manifolds, which in turn implies a diameter estimate based on the ideas from [6, 23]. We then prove a Lichnerowicz-type estimate under slightly stronger assumptions in Section 2.3. In Section 2.4 we prove a Bonnet-Myers theorem that is based on a spectral assumption of a certain Schrödinger operator. Section 3 presents all the Cheeger, Buser, and isoperimetric estimates, whereas in Section 4 we show how to bound the Kato constant under purely geometric assumptions.

Acknowledgement. G.C. was partially supported by the ANR grant CCEM-17CE40-0034 and he wants to thank the Centre Henri Lebesgue ANR-11-LABX0020-01 for creating an attractive mathematical environment. C.R. wants to thank the regional project DéfiMaths and G.C. for their hospitality during his stay in Nantes, where parts of this work had been done.

\section{A Sobolev INEQUALITY AND DiAMETER ESTIMATE UNDER SPECTRAL HYPOTHESES}

2.1. Sobolev inequality. The following is an elaboration from a result of D. Bakry [6] and revisited by I. Mondello [23].

Proposition 2.1. Let $\left(M^{n}, g\right)$ be a closed Riemannian manifold such that for some $\delta \in\left(\frac{n+1}{n+4}, 1\right)$, the operator

$$
(1-\delta) \nabla^{*} \nabla+\operatorname{Ricci}-(n-1) \operatorname{Id}
$$

is non negative.1. Then for $p_{n, \delta}=\frac{(1+\delta) n-1+\delta}{n-1-\delta}$ and $\gamma_{n, \delta}=\frac{(3+\delta)(n-\delta)}{(n-1-\delta) n(n-1)}$, we get the Sobolev inequality

$$
\forall v \in \mathcal{C}^{\infty}(M):\|v\|_{L^{p_{n, \delta}}}^{2} \leq \gamma_{n, \delta}\|d v\|_{L^{2}}^{2}+\|v\|_{L^{2}}^{2}
$$

where all norms are taken with respect to the probability measure $d \mu=\frac{\mathrm{dvol}_{g}}{\operatorname{vol}_{g}(M)}$.

$$
1_{\text {i.e. } \forall \alpha \in \mathcal{C}^{\infty}}\left(T^{*} M\right):(1-\delta) \int_{M}|\nabla \alpha|^{2}+\int_{M} \operatorname{Ricci}(\alpha, \alpha) \geq(n-1) \int_{M}|\alpha|^{2}
$$


GEOMETRIC AND SPECTRAL ESTIMATES BASED ON SPECTRAL RICCI CURVATURE ASSUMPTIONS

Proof. We procced as in [6]. For $p \in\left(2, \frac{2 n}{n-2}\right)$ and $\epsilon>0$ there is a positive function $f$ such that $\|f\|_{L^{p}}=1$ and that realizes the best constant in the inequality

$$
\forall v \in \mathcal{C}^{\infty}(M):\|v\|_{L^{p}}^{2} \leq \gamma\|d v\|_{L^{2}}^{2}+(1+\epsilon)\|v\|_{L^{2}}^{2} .
$$

The Euler-Lagrange equation for the above problem implies that $f$ solves the equation

$$
\gamma \Delta f+(1+\epsilon) f=f^{p-1}
$$

For $\alpha \in \mathbb{R}$, we define $u$ by $f=u^{\alpha}$ and the computation of Bakry leads to

$$
\begin{aligned}
(1+\epsilon) \frac{p-2}{\gamma} \int_{M}|d u|^{2} d \mu & =\int_{M}(\Delta u)^{2} d \mu \\
& +(\alpha-1)(1+\alpha(p-2)) \int_{M} \frac{|d u|^{4}}{u^{2}} d \mu \\
& -\alpha(p-1) \int_{M} \Delta u \frac{|d u|^{2}}{u} d \mu .
\end{aligned}
$$

With $A$ the traceless part of the Hessian of $u$ :

$$
A=\nabla d u+\frac{\Delta u}{n} g
$$

and the Bochner formula, we get

$$
\begin{aligned}
\int_{M}(\Delta u)^{2} d \mu & =\int_{M}|\nabla d u|^{2} d \mu+\int_{M} \operatorname{Ricci}(d u, d u) d \mu \\
& \geq(n-1) \int_{M}|d u|^{2} d \mu+\delta \int_{M}|\nabla d u|^{2} d \mu \\
& \geq(n-1) \int_{M}|d u|^{2} d \mu+\frac{\delta}{n} \int_{M}|\Delta u|^{2} d \mu+\delta \int_{M}|A|^{2} d \mu .
\end{aligned}
$$

Hence

$$
\int_{M}(\Delta u)^{2} d \mu \geq \frac{n(n-1)}{n-\delta} \int_{M}|d u|^{2} d \mu+\frac{n \delta}{n-\delta} \int_{M}|A|^{2} d \mu .
$$

To estimate the last term of (1), we integrate by parts and get

$$
\begin{aligned}
\int_{M} \Delta u \frac{|d u|^{2}}{u} d \mu= & \int_{M}\left\langle\nabla u, \nabla \frac{|d u|^{2}}{u}\right\rangle d \mu \\
& =\int_{M} 2 \frac{\nabla d u(d u, d u)}{u} d \mu-\int_{M} \frac{|d u|^{4}}{u^{2}} d \mu \\
& =-\frac{2}{n} \int_{M} \Delta u \frac{|d u|^{2}}{u} d \mu+2 \int_{M} \frac{A(d u, d u)}{u} d \mu-\int_{M} \frac{|d u|^{4}}{u^{2}} d \mu
\end{aligned}
$$

hence one gets

$$
\int_{M} \Delta u \frac{|d u|^{2}}{u} d \mu=-\frac{n}{n+2} \int_{M} \frac{|d u|^{4}}{u^{2}} d \mu+\frac{2 n}{n+2} \int_{M} \frac{A(d u, d u)}{u} d \mu .
$$


This implies

(2)

$$
\begin{aligned}
\left((1+\epsilon) \frac{p-2}{\gamma}-\frac{n(n-1)}{n-\delta}\right) \int_{M}|d u|^{2} d \mu= & \frac{n \delta}{n-\delta} \int_{M}|A|^{2} d \mu \\
& -2 n \alpha \frac{p-1}{n+2} \int_{M} \frac{A(d u, d u)}{u} d \mu \\
& C(\alpha) \int_{M} \frac{|d u|^{4}}{u^{2}} d \mu
\end{aligned}
$$

where $C(\alpha)=(\alpha-1)(1+\alpha(p-2))+\frac{n}{n+2} \alpha(p-1)$. But since Trace $A=0$, we get

$$
2 \frac{A(d u, d u)}{u}=2\left\langle A, \frac{d u \otimes d u}{u}\right\rangle=2\left\langle A, \frac{d u \otimes d u}{u}-\frac{1}{n} \frac{|d u|^{2}}{u} \mathrm{Id}\right\rangle
$$

and

$$
2\left|\frac{A(d u, d u)}{u}\right| \leq 2|A| \sqrt{\frac{n-1}{n}} \frac{|d u|^{2}}{u} \leq \lambda|A|^{2}+\frac{n-1}{n \lambda} \frac{|d u|^{4}}{u^{2}} .
$$

Chosing $\lambda$ such that

$$
\frac{n}{n-\delta}-n \alpha \frac{p-1}{n+2} \lambda=0
$$

we get

$$
\left((1+\epsilon) \frac{p-2}{\gamma}-\frac{n(n-1)}{n-\delta}\right) \int_{M}|d u|^{2} d \mu \geq F(\alpha) \int_{M} \frac{|d u|^{4}}{u^{2}} d \mu,
$$

where $F(\alpha)$ is the quadratic expression

$$
F(\alpha)=A \alpha^{2}+B \alpha-1
$$

with $A=p-2 \frac{(n-1)(p-1)^{2}(n-\delta)}{\delta(n+2)^{2}}$ and $B=2-2 \frac{p-1}{n+2}$. A little bit of arithmetic gives that

$$
\frac{B^{2}}{4}+A=\frac{n(p-1)}{n+2}\left(1+\frac{p-1}{n+2} \frac{-n+1+\delta}{\delta}\right) .
$$

This quantity is non positive if and only if

$$
0 \leq p-1 \leq \delta \frac{n+2}{n-1-\delta}
$$

Hence in that case, one can choose $\alpha$ such that $F(\alpha) \geq 0$ and we get

$$
(1+\epsilon) \frac{p-2}{\gamma} \geq \frac{n(n-1)}{n-\delta} .
$$

It is then easy to conclude. 
GEOMETRIC AND SPECTRAL ESTIMATES BASED ON SPECTRAL RICCI CURVATURE ASSUMPTION9

2.2. Diameter estimate. With the diameter estimates of Bakry-Ledoux [5], we get

Corollary 2.2. Let $\left(M^{n}, g\right)$ be a closed Riemannian manifold satisfying the hypothesis of Proposition 2.1] then

$$
\operatorname{diam}(M, g) \leq \pi C(n, \delta)
$$

Where $C(n, \delta)=\frac{\sqrt{2 p_{n, \delta} \gamma_{n, \delta}}}{p_{n, \delta}-2}=\sqrt{\frac{1-(1-\delta) \frac{n-1}{n}}{1-(1-\delta) \frac{n+3}{4}}\left(1+\frac{1-\delta}{n-1}\right)}$.

Remarks 2.3. i) If $\left(M^{n}, g\right)$ is such that for some $\delta>0$ with $1-\delta<\frac{4}{n+3}$, we have

$$
(1-\delta) \nabla^{*} \nabla+\operatorname{Ricci} \geq(n-1) k^{2},
$$

then the metric $k^{2} g$ satisfies the hypothesis of the Proposition 2.1 and hence the original metric $g$ satisfies the Sobolev type inequality

$$
\forall v \in \mathcal{C}^{\infty}(M):\|v\|_{L^{p_{n, \delta}}}^{2} \leq \kappa \gamma_{n, \delta}\|d v\|_{L^{2}}^{2}+\|v\|_{L^{2}}^{2},
$$

and hence the diameter estimate

$$
\operatorname{diam} M \leq \frac{\pi}{k} C(n, \delta) .
$$

ii) If the bottom of the spectrum of the Schrödinger operator $(1-\delta) \Delta+\rho$ is strictly positive for some $\delta>0$ with $1-\delta<\frac{4}{n+3}$, i.e., there is some $\mu>0$ with

$$
\forall v \in \mathcal{C}^{\infty}(M):(1-\delta)\|d v\|_{L^{2}}^{2}+\int_{M} \rho v^{2} \operatorname{dvol}_{g} \geq \mu\|v\|_{L^{2}},
$$

then the condition (3) is satisfied with $k=\sqrt{\mu /(n-1)}$. If this spectral condition is satisfied on $(M, g)$ then it is satisfied on any covering $\widehat{M} \rightarrow M$, but our result gives a Sobolev inequality and a diameter estimate only if $\widehat{M}$ is closed, i.e., for finite covering of $M$. We will see in the next section how such a spectral condition implies that the universal cover of $M$ has finite volume and hence that the fundamental group of $M$ is finite.

One can give a Kato-type condition that implies the condition (3): Assume that

$$
\kappa_{T}\left((\rho-\lambda)_{-}\right) \leq(1-\delta)\left(1-e^{-\beta T}\right)
$$

where $0 \leq 1-\delta<\frac{4}{n+3}$. Then we get

$$
\left\|e^{-T((1-\delta) \Delta-(\rho-\lambda)-}\right\|_{L^{\infty} \rightarrow L^{\infty}} \leq e^{\frac{\beta}{1-\delta} T},
$$

and hence

$$
(1-\delta) \Delta+\rho \geq-\frac{\beta}{1-\delta}+\lambda
$$

If moreover $\beta<(1-\delta) \lambda$, we get that the bottom of the spectrum of the Schrödinger operator $(1-\delta) \Delta+\rho$ is bounded from below by $-\frac{\beta}{1-\delta}+\lambda$. This implies the following corollary: 
Corollary 2.4. Let $\left(M^{n}, g\right)$ be a closed manifold of dimension $n \geq 3$ such that for some $\in \in\left[0, \frac{4}{n+3}\right)$ and $k, \lambda>0$ with $(n-1) k^{2}<\lambda$, we have

$$
\kappa_{T}\left((\rho-\lambda)_{-}\right) \leq \epsilon\left(1-e^{-\frac{T}{\epsilon}\left(\lambda-(n-1) k^{2}\right)}\right) .
$$

Then, the diameter of $M$ can be bounded by

$$
\operatorname{diam}(M) \leq \frac{\pi}{k} C(n, 1-\epsilon) .
$$

2.3. Lichnerowicz eigenvalue estimate. It is easy to show that with the same arguments used in the beginning of the proof of the Proposition 2.1, we get an eigenvalue estimate:

Lemma 2.5. Let $\left(M^{n}, g\right)$ be a closed manifold of dimension $n \geq 2$, such that for some $\delta \in(0,1]$ and $k>0$ the Schrödinger operator $(1-\delta) \Delta+\rho-(n-1) k^{2}$ is non negative. Then, we have

$$
\lambda_{1}(M) \geq \frac{n-1}{n-\delta} n k^{2} .
$$

We have found another method for proving a better estimate.

Proposition 2.6. Let $\left(M^{n}, g\right)$ be a closed manifold of dimension $n \geq 2$, such that for some $k>0$ the Schrödinger operator $\frac{n}{n-1} \Delta+\rho-(n-1) k^{2}$ is non negative. Then, the first eigenvalue $\lambda_{1}(M)$ satisfies

$$
\lambda_{1}(M) \geq n k^{2} .
$$

Proof. By scaling, one can assume that $k=1$. Let $\lambda>0$ be the first non-zero eigenvalue of the Laplacian with eigenfunction $\varphi$, i.e.,

$$
\Delta \varphi=\lambda \varphi \text {. }
$$

For $\alpha>0$, we define

$$
h:=r^{\alpha} \varphi \in \mathcal{C}^{\infty}((0, \infty) \times M) .
$$

The manifold $K:=(0, \infty) \times M$, is equipped with the cone metric $k=d r^{2}+r^{2} g$, such that the Laplacian $\Delta_{h}$ on $K$ has the representation

We have

$$
\Delta_{k}=-\partial_{r}^{2}-\frac{n}{r} \partial_{r}+\frac{1}{r^{2}} \Delta .
$$

$$
\Delta_{k} h=(-\alpha(\alpha-1)-n \alpha+\lambda) r^{\alpha-2} \varphi .
$$

Hence, we choose $\alpha>0$ so that

$$
\lambda:=\alpha(n-1+\alpha)
$$

then $h$ will be a harmonic function on $(K, k)$. Note that $\alpha \geq 1$ is equivalent to $\lambda \geq n$.

The Bochner formula on $K$ and the refined Kato inequality yield (see for instance [8, Lemma 3.4])

$$
\Delta_{k}\left|d_{k} h\right|^{\frac{n-1}{n}} \leq \frac{n-1}{n} \rho^{K}\left|d_{k} h\right|^{\frac{n-1}{n}},
$$


where $d_{k}$ denotes the differential and $\rho^{K}$ is the lowest eigenvalue of the Ricci tensor on $K$. Now, the norm on the cotangent bundle of $d_{k} h$ is

$$
\left|d_{k} h\right|^{2}=r^{2 \alpha-2}\left[\alpha^{2} \varphi^{2}+|d \varphi|^{2}\right]
$$

such that the second factor is independent of $r$. For

$$
\psi:=\left[\alpha^{2} \varphi^{2}+|d \varphi|^{2}\right]^{\frac{n-1}{2 n}},
$$

we have

$$
-(\alpha-1) \frac{n-1}{n}\left[(\alpha-1) \frac{n-1}{n}+n-1\right] \psi+\Delta \psi+\frac{n-1}{n} V \psi \leq 0,
$$

where $V(x)$ is the lowest eigenvalue of the Ricci curvature at $(1, x)$ in $(K, k)$. A short calculation shows that $V(x)=\rho(x)-(n-1)$. Hence, one gets

$$
\Delta \psi+\frac{n-1}{n} V \psi \leq(\alpha-1) \frac{n-1}{n}\left[(\alpha-1) \frac{n-1}{n}+n-1\right] \psi .
$$

If we let

$$
c(\alpha):=(\alpha-1) \frac{n-1}{n}\left[(\alpha-1) \frac{n-1}{n}+n-1\right],
$$

then the above inequality (4) implies that the bottom of the spectrum of the operator $\Delta+\frac{n-1}{n} V$ is smaller than $c(\alpha)$. From our assumption, one gets $c(\alpha) \geq 0$, that is to say $\alpha \geq 1$.

Corollary 2.7. Let $\left(M^{n}, g\right)$ be a closed manifold of dimension $n \geq 3$ such that for some $k, \lambda>0$ with $\lambda>(n-1) k^{2}$ :

$$
\kappa_{T}\left((\rho-\lambda)_{-}\right) \leq \frac{n}{n-1}\left(1-e^{-T \frac{n-1}{n}\left(\lambda-(n-1) k^{2}\right)}\right) .
$$

Then, $\lambda_{1}(M)$ satisfies

$$
\lambda_{1}(M) \geq n k^{2} .
$$

The Lichnerowicz-type estimate above also yields an analogue result for integral curvature bounds by estimating the norm of the heat semigroup as it was done in, e.g., [33, 31, 18, 17].

\subsection{A Bonnet-Myers's theorem under a spectral hypothesis.}

Theorem 2.8. If $\left(M^{n}, g\right)$ is a closed connected Riemannian manifold such that for some positive $\epsilon \leq 1 /(n-2)$, the bottom of the spectrum of the Schrödinger operator

$$
\epsilon \Delta+\rho
$$

is positive then $\pi_{1}(M)$ is finite.

Proof. The main argument in the proof is in fact a modification of one part of the proof of the theorem in [11]. We are going to show that the universal cover $\pi: \widetilde{M} \rightarrow M$ has finite volume, hence the fundamental group is finite.

We first remark that if the bottom of the spectrum of the Schrödinger operator $(n-2) \Delta+\rho$ is positive, then by continuity there is some positive $\epsilon<1 /(n-2)$, 
such that the bottom of the spectrum of the Schrödinger operator $\epsilon \Delta+\rho$ is positive: there is a positive constant $\mu$ such that

$$
\forall v \in \mathcal{C}_{0}^{\infty}(M): \epsilon \int_{M}|d v|^{2}+\int 4_{M} \rho v^{2} \geq \mu \int_{M} v^{2}
$$

Let $\varphi: M \rightarrow \mathbb{R}$ be a positive eigenfunction of the Schrödinger operator $\epsilon \Delta+\rho$ associated to the bottom of the spectrum. We have $\epsilon \Delta \varphi+\rho \varphi=\lambda \varphi$ with $\lambda \geq \mu$. Hence on $\widetilde{M}$, one has for $\widetilde{\varphi}=\varphi \circ \pi$ :

$$
\epsilon \Delta_{\widetilde{M}} \widetilde{\varphi}+\rho \widetilde{\varphi}=\lambda \widetilde{\varphi}
$$

By a principle due to W.F. Moss and J. Piepenbrink, and D. Fisher-Colbrie and R. Schoen, $([24,16]$ or [30, lemma 3.10]), that is in fact based on an old result of J. Barta ([7]), we know that $\left(\widetilde{M}, \tilde{g}=\pi^{*} g\right)$ also satisfies

$$
\forall v \in \mathcal{C}_{0}^{\infty}(\widetilde{M}): \epsilon \int_{\widetilde{M}}|d v|^{2}+\int_{\widetilde{M}} \rho v^{2} \geq \mu \int_{\widetilde{M}} v^{2}
$$

With the Bochner formula and the Kato inequality, we get that the spectrum of the Hodge-deRham Laplacian on one-forms is positive:

$$
\operatorname{spec} \Delta_{1} \subset[\mu,+\infty) \text {. }
$$

Indeed, if $\alpha \in \mathcal{C}_{0}^{\infty}\left(T^{*} \widetilde{M}\right)$ then

$$
\begin{aligned}
\left\langle\Delta_{1} \alpha, \alpha\right\rangle & =\int_{\widetilde{M}}|\nabla \alpha|^{2}+\operatorname{Ricci}(\alpha, \alpha) \\
& \geq\left.\int_{\widetilde{M}}|d| \alpha\right|^{2}+\rho|\alpha|^{2} \\
& \geq \mu \int_{\widetilde{M}}|\alpha|^{2} .
\end{aligned}
$$

Because one always has spec $\Delta \subset\{0\} \cup \operatorname{spec} \Delta_{1}$, one deduces that the spectrum of the Laplacian $\Delta$ on functions has a spectral gap:

$$
\operatorname{spec} \Delta \subset\{0\} \cup[\mu,+\infty) \text {. }
$$

Hence either 0 is an eigenvalue for the Laplacian on $\widetilde{M}$ and this means that the volume of $\tilde{M}$ is finite ${ }^{2}$ or the bottom of the spectrum of the Laplacian is positive. It is classical that the first case implies that $\pi_{1}(M)$ is finite. We are going to show that the second case can not occur.

We are arguing by contradiction and we suppose that $\lambda_{0}(\widetilde{M})>0$. $\widetilde{M}$ being a covering of a compact Riemannian manifold, it satisfies the local Sobolev inequality :

$$
\forall v \in \mathcal{C}_{0}^{\infty}(\widetilde{M}):\|v\|_{L^{\frac{2 n}{n-2}}}^{2} \leq C\left(\|d v\|_{L^{2}}^{2}+\|d v\|_{L^{2}}^{2}\right)
$$

\footnotetext{
${ }^{2}$ Indeed, if $f$ is an eigenfunction of the Laplace operator associated to the eigenvalue 0 , then $\Delta f=0$ and $d f \in L^{2}$ hence by definition of the domain of the Laplace operator, one can integrate by parts and gets

$$
\int_{\widetilde{M}}|d f|^{2}=\int_{\widetilde{M}} f \Delta f=0 .
$$
}

Hence $f$ is constant. 
GEOMETRIC AND SPECTRAL ESTIMATES BASED ON SPECTRAL RICCI CURVATURE ASSUMPTIONS

Using the spectral gap, we get the Euclidean Sobolev inequality:

$$
\forall v \in \mathcal{C}_{0}^{\infty}(\widetilde{M}):\|v\|_{L^{\frac{2 n}{n-2}}}^{2} \leq C\left(1+\lambda_{0}(\widetilde{M})^{-1}\right)\|d v\|_{L^{2}}^{2}
$$

In particular, $(\widetilde{M}, \tilde{g})$ is non-parabolic and there is a unique positive minimal Green kernel $G(x, y)$. Let $o \in \widetilde{M}$ be a fixed point and define $b: M \rightarrow \mathbb{R}_{+}$by

$$
b^{2-n}=c_{n} G(o, x) \text {, }
$$

where $c_{n}$ is chosen so that $b(x) \simeq d(o, x)$ as $x \rightarrow o$. The Sobolev inequality implies that there is a positive constant $c$ such that

$$
c d_{\tilde{g}}(o, x) \leq b(x) .
$$

In particular, $\lim _{x \rightarrow \infty} b(x)=+\infty$. According to T.H. Colding ([13]), for any $\alpha \geq(n-2) /(n-1)$, we have

$$
\Delta \frac{|d b|^{\alpha}}{b^{n-2}}+\alpha \rho \frac{|d b|^{\alpha}}{b^{n-2}} \leq 0 \text { weakly on } \widetilde{M} \backslash\{o\} .
$$

Our hypothesis implies that for any $\alpha \in[0,1 / \epsilon]$, the Schrödinger operator $\Delta+\alpha \rho$ has a spectral gap, hence it has a unique minimal positive Green kernel $G_{\alpha}$. We let $g_{\alpha}(x)=G_{\alpha}(o, x) / c_{n}$. It is then classical (see [11, subsection 2.6.2]) that

$$
\int_{\widetilde{M} \backslash B(o, 1)}\left|d g_{\alpha}\right|^{2}<\infty
$$

Then the spectral gap

$$
\forall \varphi \in \mathcal{C}^{\infty}(\widetilde{M}): \int_{\widetilde{M}} \varphi^{2} \leq \int_{\widetilde{M}}|d \varphi|^{2}+\alpha \rho \varphi^{2}
$$

and the proof of the above estimate (5) implies that

$$
\int_{\widetilde{M} \backslash B(o, 1)} G_{\alpha}^{2}<\infty
$$

Note that with the local Sobolev inequality, the classical De Giorgi-Nash-Moser iteration scheme (see [19, theorem 8.17] or [23, Lemma 1.5]) implies that a weak non-negative $W_{\text {loc }}^{1,2}$ solution of

$$
\Delta \varphi \leq \Lambda \varphi
$$

is in fact locally bounded: for any $p \geq 1$, there is a constant $C$ depending only on $r, \Lambda$ and the local Sobolev constant so that

$$
\sup _{y \in B(x, r)} \varphi(y) \leq C\left(\int_{B(x, 2 r)} \varphi^{p}\right)^{\frac{1}{p}} .
$$

$(\widetilde{M}, \tilde{g})$ being a covering of a compact Riemannian manifold, the Ricci curvature is bounded from below: $\operatorname{Ricci}_{\tilde{g}} \geq-(n-1) k^{2}$ so that $-\rho \leq(n-1) k^{2}$. Hence we get the following elliptic estimate: for any $\alpha$ and $p \geq 1$ there is a constant $C$ such that if a non negative function $\varphi \in W_{l o c}^{1,2}$ satisfies

$$
\Delta \varphi+\alpha \rho \varphi \leq 0 \text { weakly on } B(x, 2)
$$


then

$$
\sup _{y \in B(x, 1)} \varphi(y) \leq C\left(\int_{B(x, 2)} \varphi^{p}\right)^{\frac{1}{p}} .
$$

In particular, we get (when $\alpha \in[0,1 / \epsilon]$ ) that $\lim _{x \rightarrow \infty} g_{\alpha}(x)=0$, and we have the equivalence

With the estimate (5), one gets

$$
\frac{|d b|^{\alpha}}{b^{n-2}} \leq g_{\alpha} \Longleftrightarrow \lim _{x \rightarrow \infty} \frac{|d b|^{\alpha}}{b^{n-2}}=0 .
$$

$$
\int_{\widetilde{M} \backslash B(o, 1)}\left|d_{x} G(o, x)\right|^{2} \operatorname{dvol}_{\tilde{g}}(x)<\infty,
$$

we deduce that for $\alpha_{0}:=\frac{n-2}{n-1}$, we have

$$
\lim _{x \rightarrow \infty} \frac{|d b|^{\alpha_{0}}}{b^{n-2}}=0
$$

and hence

$$
\frac{|d b|^{\alpha_{0}}}{b^{n-2}} \leq g_{\alpha_{0}}
$$

Our main tool is the universal Hardy inequality ([[10]): $\forall \psi \in \mathcal{C}_{0}^{\infty}(\widetilde{M})$ :

$$
\frac{(n-2)^{2}}{4} \int_{\widetilde{M}} \frac{|d b|^{2}}{b^{2}} \psi^{2} \operatorname{dvol}_{\tilde{g}} \leq \int_{\widetilde{M}}|d \psi|^{2} \operatorname{dvol}_{\tilde{g}} .
$$

The Schrödinger operator $\epsilon \Delta+\rho$ is positive hence for any $\alpha \in[0, n-2]$, we have the following Hardy type inequality: $\forall \psi \in \mathcal{C}_{0}^{\infty}(\widetilde{M})$ :

$$
c \int_{\widetilde{M}} \frac{|d b|^{2}}{b^{2}} \psi^{2} \operatorname{dvol}_{\tilde{g}} \leq \int_{\widetilde{M}}\left[|d \psi|^{2}+\alpha \rho \psi^{2}\right] \operatorname{dvol}_{\tilde{g}} .
$$

When $\alpha \in\left[\alpha_{0}, n-2\right]$, using the function $\psi=\xi \frac{|d b|^{\alpha}}{b^{n-2}}$ where $\xi$ is a Lipschitz function with compact support in $\widetilde{M} \backslash\{o\}$ one gets

$$
c \int_{\widetilde{M}} \frac{|d b|^{2+2 \alpha}}{b^{2(n-1)}} \xi^{2} \operatorname{dvol}_{\tilde{g}} \leq \int_{\widetilde{M}}|d \xi|^{2} \frac{|d b|^{2 p}}{b^{2(n-2)}} \mathrm{dv}_{g} .
$$

Assume that for some $\alpha \in\left[\alpha_{0}, n-2\right]$, we have

$$
\frac{|d b|^{\alpha}(x)}{b^{n-2}(x)} \leq g_{\alpha}(o, x) \text {. }
$$

Then we get

$$
\forall \xi \in \mathcal{C}_{0}^{\infty}(\widetilde{M} \backslash\{o\}): c \int_{\widetilde{M}} \frac{|d b|^{2+2 \alpha}}{b^{2(n-1)}} \xi^{2} \operatorname{dvol}_{\tilde{g}} \leq \int_{\widetilde{M}}|d \xi|^{2} g_{\alpha}^{2} \operatorname{dvol}_{\tilde{g}}
$$

According to [11, Proposition 2.21], if $\xi$ is a smooth function with support in $\widetilde{M} \backslash$ $B(o, 1 / 2)$ that is identitically 1 on $\widetilde{M} \backslash B(o, 1)$, then there is a sequence of smooth functions $\left(\xi_{\ell}\right)_{\ell}$ with compact support in $\widetilde{M} \backslash\{o\}$ such that

$$
\lim _{\ell} \xi_{\ell}=\xi \text { uniformly on compact subset of } \widetilde{M} \text {. }
$$


and

$$
\lim _{\ell} \int_{\widetilde{M}}\left|d \xi_{\ell}\right|^{2} g_{\alpha}^{2} \mathrm{dv}_{g}=\int_{\widetilde{M}}|d \xi|^{2} g_{\alpha}^{2} \mathrm{~d} v_{g}
$$

In particular, we get

$$
\int_{\widetilde{M} \backslash B(o, 1)} \frac{|d b|^{2+2 \alpha}}{b^{2(n-1)}} \mathrm{dv}_{g}<\infty
$$

If $\alpha^{\prime}=(1+\alpha) \frac{n-2}{n-1}$ one gets

$$
\int_{M \backslash B(o, 1)}\left(\frac{|d b|^{\alpha^{\prime}}}{b^{n-2}}\right)^{2 \frac{n-1}{n-2}} \mathrm{dv}_{g}<\infty,
$$

hence,

$$
\lim _{x \rightarrow \infty} \frac{|d b|^{\alpha^{\prime}}(x)}{b^{n-2}(x)}=0
$$

and

$$
\frac{|d b|^{\alpha^{\prime}}(x)}{b^{n-2}(x)} \leq g_{\alpha^{\prime}}
$$

For $\alpha_{k}=(n-2)-\left(\frac{n-2}{n-1}\right)^{k}\left(n-2-\alpha_{0}\right)=\left(1+\alpha_{k-1}\right) \frac{n-2}{n-1}$, our argumentation yields that for all $k \in \mathbb{N}$ :

$$
\frac{|d b|^{\alpha_{k}}(x)}{b^{n-2}(x)} \leq g_{\alpha_{k}}(o, x) .
$$

Hence letting $k \rightarrow \infty$, we obtain the following estimate on the log derivative of the Green kernel:

And in particular

$$
\frac{|d b|^{n-2}(x)}{b^{n-2}(x)} \leq g_{n-2}(x) .
$$

$$
\lim _{x \rightarrow \infty} \frac{|d b|(x)}{b(x)}=0 .
$$

We are going to show that this implies that $\lambda_{0}(\widetilde{M})=0$, hence a contradiction. The Green formula implies that if $t$ is a regular value of $b$, then 3

$$
\int_{\{b=t\}}|d b|=\sigma_{n-1} t^{n-1},
$$

so that

$$
\int_{\{b \leq R\}}|d b|^{2}=\omega_{n} R^{n}
$$

and

$$
\int_{\{R \leq 4 b \leq 3 R\}}|d b|^{2}=\omega_{n}\left(3^{n}-1\right) R^{n} .
$$

\footnotetext{
$3_{\text {with }} \sigma_{n-1}=\operatorname{vol} \mathbb{S}^{n-1}$.

${ }^{4}$ With $\omega_{n}=\operatorname{vol} \mathbb{B}^{n}$.
} 
Let $\Omega_{R}:=\{b \leq R\}$ and $\varphi_{R}=(R-b)_{+}$. We have that $\varphi_{R}$ has support in $\Omega_{R}$ and

$$
\int_{\Omega_{R}}\left|d \varphi_{R}\right|^{2}=\omega_{n} R^{n}=\frac{1}{3^{n}-1} \int_{\{R \leq 4 b \leq 3 R\}}|d b|^{2} .
$$

But

$$
\int_{\Omega_{R}} \varphi_{R}^{2} \geq \int_{\{R \leq 4 b \leq 3 R\}} \varphi_{R}^{2} \geq \frac{1}{9} \int_{\{R \leq 4 b \leq 3 R\}} b^{2} .
$$

We have shown that

$$
\lim _{x \rightarrow \infty} \frac{|d b|(x)}{b(x)}=0
$$

hence

$$
\lim _{R \rightarrow \infty} \frac{\int_{\Omega_{R}}\left|d \varphi_{R}\right|^{2}}{\int_{\Omega_{R}}\left|\varphi_{R}\right|^{2}}=0
$$

hence $\lambda_{0}(\widetilde{M})=0$.

\section{BUSER INEQUALITY AND ISOPERIMETRIC ESTIMATES}

We are going to use an idea from M. Ledoux [22] in order to obtain isoperimetric inequalities.

3.1. Buser inequality. To apply Ledoux's technique, we recall the gradient estimates for positive solutions of the heat equation from [32, 11]:

Proposition 3.1. Let $\left(M^{n}, g\right)$ be a closed Riemannian manifold of dimension $n \geq$ 2 such that for some $T>0$ :

$$
\kappa_{T}\left(\rho_{-}\right) \leq \frac{1}{16 n}
$$

If $u:[0, T] \times M \rightarrow \mathbb{R}_{+}$be a positive solution of the heat equation

$$
\partial_{t} u=-\Delta u
$$

then, we have

$$
e^{-2} \frac{|\nabla u|^{2}}{u^{2}}-\frac{\partial_{t} u}{u} \leq \frac{e^{2} n}{2 t}, \quad \forall t \in(0, T) .
$$

From Proposition 3.1, we can deduce

Corollary 3.2. Under the assumptions of Proposition 3.1 there is an explicit constant $c_{n}$ depending only on the dimension, such that for any $t \in(0, T]$ :

$$
\left\|\Delta P_{t}\right\|_{L^{\infty} \rightarrow L^{\infty}} \leq \frac{c_{n}}{t}
$$

and

$$
\left\|\nabla P_{t}\right\|_{L^{\infty} \rightarrow L^{\infty}} \leq \frac{c_{n}}{\sqrt{t}}
$$


GEOMETRIC AND SPECTRAL ESTIMATES BASED ON SPECTRAL RICCI CURVATURE ASSUMPTION\$

Proof. Let $f \in L^{1}(M)$ be a non negative initial value for the heat equation with $u=P_{t} f$. Note that

$$
(\Delta u)_{+}=\left(-\partial_{t} u\right)_{+} \leq \frac{e^{2} n}{2 t} u
$$

By Stokes theorem, we have $\int \Delta u=0$. Furthermore, we know $P_{t} 1=1$ and $\int P_{t} f=\int f$. Hence,

$$
\left\|\Delta P_{t} f\right\|_{L^{1}}=2\left\|\left(\Delta P_{t} f\right)_{+}\right\|_{L^{1}} \leq \frac{e^{2} n}{t}\|u\|_{L^{1}}=\frac{e^{2} n}{t}\|f\|_{L^{1}}
$$

We easily get

$$
\left\|\Delta P_{t}\right\|_{L^{1} \rightarrow L^{1}} \leq \frac{e^{2} n}{t}
$$

By duality and self-adjointness of the operator $\Delta P_{t}$ we obtain the first assertion.

Let $f \in L^{\infty}(M)$ be a non negative initial value for the heat equation and let $u=P_{t} f$. We have

$$
|\nabla u|^{2} \leq e^{2}\left(\frac{e^{2} n}{2 t} u^{2}+u|\Delta u|\right)
$$

and, therefore,

$$
\begin{aligned}
\|\nabla u\|_{L^{\infty}}^{2} & \leq e^{2}\left(\frac{e^{2} n}{2 t}+\frac{e^{2} n}{t}\right)\|u\|_{L^{\infty}}^{2} \\
& \leq \frac{3 e^{4} n}{2 t}\|f\|_{L^{\infty}}^{2} .
\end{aligned}
$$

In general, for $f \in L^{\infty}(M)$, we get

$$
\left\|\nabla P_{t} f\right\|_{L^{\infty}} \leq \sqrt{\frac{14 n}{t}}\|f\|_{L^{\infty}}
$$

The above proposition implies the following crucial estimate.

Proposition 3.3. Under the assumptions of Proposition 3.1 there is a constant5 $c_{n}$, such that for any $t \in(0, T]$, we have

$$
\forall f \in W^{1,1}(M):\left\|f-P_{t} f\right\|_{L^{1}} \leq c_{n} \sqrt{t}\|d f\|_{L^{1}} .
$$

Proof. We have

$$
f-P_{t} f=\int_{0}^{t} \Delta e^{-s \Delta} f d s .
$$

\footnotetext{
${ }^{5}$ One can choose $c_{n}=6 \sqrt{n}$.
} 
For any $g \in L^{\infty}$, the above yields

$$
\begin{aligned}
\int_{M} g \Delta e^{-s \Delta} f \mathrm{dvol} & =\int_{M} g \Delta P_{s} f \mathrm{dvol} \\
& =\int_{M}\left\langle\nabla P_{t} g, \nabla f\right\rangle \mathrm{dvol} \\
& \leq \sqrt{\frac{14 n}{t}}\|\nabla f\|_{L^{1}}\|g\|_{L^{\infty}}
\end{aligned}
$$

Hence, we get

$$
\left\|\Delta e^{-s \Delta} f\right\|_{L^{1}} \leq \sqrt{\frac{14 n}{t}}\|\nabla f\|_{L^{1}}
$$

what implies

$$
\left\|f-P_{t} f\right\|_{L^{1}} \leq c_{n} \sqrt{t}\|d f\|_{L^{1}}
$$

with $c_{n}=6 \sqrt{n}$.

Now, we can obtain the following estimate relating the Cheeger constant and the first non-zero eigenvalue of the Laplacian on $M$.

Theorem 3.4. Let $\left(M^{n}, g\right)$ be a closed Riemannian manifold of dimension $n \geq 2$ such that for some $T>0$, we have

$$
\kappa_{T}\left(\rho_{-}\right) \leq \frac{1}{16 n}
$$

There is a explicit constant $c_{n}$ such that if $\lambda_{1}$ is the first non-zero eigenvalue of the Laplacian on $M$ satisfies

$$
\lambda_{1}(M) \leq c_{n}\left(\frac{1}{\sqrt{T}} \mathrm{~h}(M)+\mathrm{h}^{2}(M)\right) .
$$

Proof. We apply the above inequality to the characteristic function $\chi_{\Omega}$ of $\Omega$. From the fact that $P_{t}\left(\chi_{\Omega}\right) \leq 1$ we get

$$
\begin{aligned}
\int_{M}\left|\chi_{\Omega}-P_{t}\left(\chi_{\Omega}\right)\right| \mathrm{dvol} & =\int_{\Omega}\left|\chi_{\Omega}-P_{t}\left(\chi_{\Omega}\right)\right| \mathrm{dvol}+\int_{M \backslash \Omega} P_{t}\left(\chi_{\Omega}\right) \mathrm{dvol} \\
& \int_{\Omega}\left(\chi_{\Omega}-P_{t}\left(\chi_{\Omega}\right)\right) \mathrm{dvol}+\int_{M \backslash \Omega} P_{t}\left(\chi_{\Omega}\right) \mathrm{dvol} .
\end{aligned}
$$

Since $\int_{\Omega} P_{t}\left(\chi_{\Omega}\right) \mathrm{dvol}=\left\|P_{t / 2} \chi_{\Omega}\right\|_{L^{2}}^{2}$ and

$$
\int_{M \backslash \Omega} P_{t}\left(\chi_{\Omega}\right) \mathrm{dvol}=\left\langle P_{t}\left(\chi_{M \backslash \Omega}\right), \chi_{\Omega}\right\rangle=\left\langle 1-P_{t}\left(\chi_{\Omega}\right), \chi_{\Omega}\right\rangle=\operatorname{vol} \Omega-\left\|P_{t / 2} \chi_{\Omega}\right\|_{L^{2}}^{2},
$$

we have

$$
\int_{M}\left|\chi_{\Omega}-P_{t}\left(\chi_{\Omega}\right)\right| \mathrm{dvol}=2\left(\operatorname{vol} \Omega-\left\|P_{t / 2} \chi_{\Omega}\right\|_{L^{2}}^{2}\right)
$$


We can decompose $\chi_{\Omega}=\frac{\operatorname{vol} \Omega}{\operatorname{vol} M}+f$ where $\int_{M} f \mathrm{dvol}=0$ and get

$$
\left\|P_{t / 2} \chi_{\Omega}\right\|_{L^{2}}^{2} \leq \frac{(\operatorname{vol} \Omega)^{2}}{\operatorname{vol} M}+e^{-\lambda_{1} t}\left(\operatorname{vol} \Omega-\frac{(\operatorname{vol} \Omega)^{2}}{\operatorname{vol} M}\right)
$$

This yields

$$
2 \operatorname{vol} \Omega\left(1-\frac{\operatorname{vol} \Omega}{\operatorname{vol} M}\right)\left(1-e^{-\lambda_{1} t}\right) \leq c_{n} \sqrt{t} \operatorname{vol} \partial \Omega
$$

and we get for any $t \in[0, T]$ that

$$
\left(1-e^{-\lambda_{1} t}\right) \leq c_{n} \sqrt{t} \mathrm{~h}(M) .
$$

If $T \lambda_{1} \geq 1$, then we choose $t=1 / \lambda_{1}$ and get

$$
\sqrt{\lambda_{1}} \leq 3 c_{n} \mathrm{~h}(M)
$$

In the case where $T \lambda_{1} \leq 1$, we choose $t=T$ and from the inequality $2\left(1-e^{-x}\right) \geq$ $x$ for $x \in(0,1)$, we get

$$
\lambda_{1} \sqrt{T} \leq 2 c_{n} \mathrm{~h}(M) .
$$

It is now easy to get the announced result.

3.2. An estimate of the Cheeger constant. The following corollary is an easy consequence of Theorem 3.4

Corollary 3.5. Let $\left(M^{n}, g\right)$ be a closed Riemannian manifold of dimension $n \geq 2$ and diameter at most $D>0$. There is a constant $c_{n} \geq 1$ such that if $T$ is the first positive time such that

$$
\kappa_{T}\left(\rho_{-}\right)=\frac{1}{16 n}
$$

and if we define $\xi:=\xi(M, g)=D / \sqrt{T}$, then

$$
\frac{c_{n}^{-1-\xi}}{D} \leq \mathrm{h}(M)
$$

Proof. As a matter of fact, with the eigenvalue estimate given in [11, Theorem3.6], we know that

$$
\frac{c_{n}^{-1-\xi}}{D^{2}} \leq \lambda_{1}
$$

Therefore, we have

$$
\frac{c_{n}^{-1-\xi}}{D^{2}} \leq c_{n}\left(\frac{\xi}{D} \mathrm{~h}(M)+\mathrm{h}^{2}(M)\right) .
$$

Hence either $\frac{1}{D} \leq h(M)$ or

$$
\frac{c_{n}^{-1-\xi}}{D^{2}} \leq c_{n} \frac{1+\xi}{D} \mathrm{~h}(M) \leq c_{n} \frac{e^{\xi}}{D} \mathrm{~h}(M) .
$$


3.3. Isoperimetric inequalities. From the results above, we can now obtain isoperimetric inequalities.

Theorem 3.6. Let $\left(M^{n}, g\right)$ be a closed Riemannian manifold of dimension $n \geq 2$ and diameter at most $D>0$. Assume one of the following:

i) $\kappa_{T}\left(\rho_{-}\right) \leq \frac{1}{16 n}$

ii) or that for some $p>1$, we have for some $\mathbf{I}>0$ that $D^{2 p-2} \kappa_{T}\left(\rho_{-}^{p}\right) \leq \mathbf{I}^{p}$.

In the first case let $\xi:=\xi(M, g)=D / \sqrt{T}$ and $\nu=n e^{8 \sqrt{n \kappa_{T}\left(\rho_{-}\right)}}$and in the second case let $\xi=\max \left\{\frac{D}{\sqrt{T}},(16 n \mathbf{I})^{\frac{p}{2 p-2}}\right\}$, and $\nu=n$.

Then we have

$$
1 \leq c_{n}^{1+\xi} \operatorname{vol}(M)^{\frac{1}{\nu}} D I_{\nu}(M) .
$$

Proof. From [11, Proposition 3.7 and 3.14], we get for any $t \in\left[0, D^{2}\right]$ :

$$
\left\|P_{t}\right\|_{L^{1} \rightarrow L^{\infty}} \leq \frac{c_{n}^{1+\xi}}{\operatorname{vol} M} \frac{D^{\nu}}{t^{\frac{\nu}{2}}} .
$$

Let $\Omega \subset M$ with smooth boundary and $\operatorname{vol} \Omega \leq \frac{1}{2}$ vol $M$. Then, we have

$$
\operatorname{vol} \Omega \leq \operatorname{vol}\left\{x \in M,\left|\chi_{\Omega}-P_{t}\left(\chi_{\Omega}\right)\right|>1 / 2\right\}+\operatorname{vol}\left\{x \in M, P_{t}\left(\chi_{\Omega}\right)>1 / 2\right\} .
$$

Furthermore,

$$
\left\|P_{t}\left(\chi_{\Omega}\right)\right\|_{L^{\infty}} \leq \frac{c_{n}^{1+\xi} \operatorname{vol} \Omega}{\operatorname{vol} M} \frac{D^{\nu}}{t^{\frac{\nu}{2}}}
$$

Hence if

$$
\frac{c_{n}^{1+\xi} \operatorname{vol} \Omega}{\operatorname{vol} M} \leq \frac{1}{4}
$$

one can choose $t \in\left[0, D^{2}\right]$ such that

$$
\frac{c_{n}^{1+\xi} \operatorname{vol} \Omega}{\operatorname{vol} M} \frac{D^{\nu}}{t^{\frac{\nu}{2}}}=\frac{1}{4}
$$

such that

$$
\operatorname{vol} \Omega \leq 2\left\|\chi_{\Omega}-P_{t}\left(\chi_{\Omega}\right)\right\|_{L^{1}} \leq c_{n} \sqrt{t} \operatorname{vol} \partial \Omega \text {. }
$$

A little bit of arithemetics then implies the result when $\frac{c_{n}^{1+\xi} \operatorname{vol} \Omega}{\operatorname{vol} M} \leq \frac{1}{4}$. When $\frac{c_{n}^{1+\xi} \operatorname{vol} \Omega}{\operatorname{vol} M} \geq \frac{1}{4}$, we use the estimate on the Cheeger constant to conclude.

\section{An estimate of the Kato COnstant}

Let $\left(M^{n}, g\right)$ be a complete Riemannian manifold. We assume that it satisfies the following volume doubling condition and heat kernel estimates at scale $R$ : there are positive constants $\theta, \nu, C$ such that

$\left(\mathrm{D}_{R}\right) \quad \forall x \in M, 0 \leq r \leq \rho \leq R: \frac{\operatorname{vol} B(x, \rho)}{\operatorname{vol} B(x, r)} \leq \theta\left(\frac{\rho}{r}\right)^{\nu}$ 
GEOMETRIC AND SPECTRAL ESTIMATES BASED ON SPECTRAL RICCI CURVATURE ASSUMPTION\$

$$
\left(\mathrm{UE}_{R}\right) \quad \forall x, y \in M, t \in\left(0, R^{2}\right]: H(t, x, y) \leq \frac{C}{\operatorname{vol} B(x, \sqrt{t})} e^{-\frac{d^{2}(x, y)}{5 t}} .
$$

Recall that if $q: M \rightarrow \mathbb{R}_{+}$, is locally integrable then its parabolic Kato constant at times $T$ is given by

$$
\kappa_{T}(q)=\sup _{x \in M} \int_{0}^{T} \int_{M} H(t, x, y) q(y) \operatorname{dvol}_{g}(y) d t
$$

We know that if $\kappa_{T}(q) \leq 1-e^{-\beta T}$ for some $\beta>0$, then

$$
\forall t>0: \quad\left\|e^{-t(\Delta-q)}\right\|_{L^{\infty} \rightarrow L^{\infty}} \leq e^{\beta t},
$$

and in particular the Schrödinger operator $\Delta-q$ is bounded from below by $-\beta$ :

$$
\forall v \in \mathcal{C}_{0}^{\infty}(M): \int_{M}\left[|d v|^{2}-q v^{2}\right] \mathrm{dvol}_{g} \geq-\beta \int_{M} v^{2} \mathrm{dvol}_{g} .
$$

Proposition 4.1. There is a constant $\lambda$ depending only on $\theta, \nu$ and $C$ such that if

$$
\lim _{r \rightarrow+\infty} e^{-\frac{r^{2}}{R 2}} \int_{B(x, r)} q=0
$$

then

$$
\kappa_{R^{2}}(q) \leq \lambda \sup _{x} \int_{0}^{\infty} r e^{-\frac{r^{2}}{6 R^{2}}}\left(f_{B(x, r)} q\right) d r .
$$

Proof. We introduce the convenient notations :

$$
V(x, r)=\operatorname{vol} B(x, r) \text { and } Q(x, r)=\int_{B(x, r)} q
$$

so that

$$
f_{B(x, r)} q=\frac{Q(x, r)}{V(x, r)} .
$$

Notice that $r \mapsto Q(x, r)$ is a non-decreasing function so that we can consider the Riemann-Stieljes measure $d Q(x, r)$. Using the estimate on the heat kernel we have

$$
\int_{0}^{R^{2}} \int_{M} H(t, x, y) q(y) \operatorname{dvol}_{g}(y) d t \leq C \int_{\left[0, R^{2}\right] \times[0, \infty)} \frac{e^{-\frac{r^{2}}{5 t}}}{V(x, \sqrt{t})} d Q(x, r) d t .
$$

The condition (8) implies that we can integrate by parts:

$$
\int_{\left[0, R^{2}\right] \times[0, \infty)} \frac{e^{-\frac{r^{2}}{5 t}}}{V(x, \sqrt{t})} d Q(x, r) d t=\int_{\left[0, R^{2}\right] \times[0, \infty)} \frac{2 r e^{-\frac{r^{2}}{5 t}}}{5 t V(x, \sqrt{t})} Q(x, r) d t d r .
$$

We are going to split this integral in 3 parts corresponding to the domains $\{r \geq R\}$ $\{\sqrt{t} \leq r \leq R\}$ and $\{r \leq \sqrt{t} \leq R\}$.

Concerning the first part, when $r \geq R$, we get

$$
\int_{0}^{R^{2}} \frac{e^{-\frac{r^{2}}{5 t}}}{t V(x, \sqrt{t})} d t \leq \theta \frac{R^{\nu}}{V(x, R)} \int_{0}^{R^{2}} \frac{e^{-\frac{r^{2}}{5 t}}}{t^{\frac{\nu}{2}+1}} d t .
$$


Using $\xi=r^{2} /(5 t)$, we obtain

$$
\int_{0}^{R^{2}} \frac{e^{-\frac{r^{2}}{5 t}}}{t^{\frac{\nu}{2}+1}} d t \leq \frac{5^{\nu}}{r^{\nu}} \int_{\frac{r^{2}}{5 R^{2}}}^{\infty} e^{-\xi} \xi^{\frac{\nu}{2}-1} d \xi
$$

Now there is a constant $c(\nu)$ such that when $A \geq 1 / 5$ :

$$
\int_{A}^{\infty} e^{-\xi} \xi^{\frac{\nu}{2}-1} d \xi \leq c(\nu) e^{-A} A^{\frac{\nu}{2}-1}
$$

and we get

$$
\int_{0}^{R^{2}} \frac{e^{-\frac{r^{2}}{5 t}}}{t V(x, \sqrt{t})} d t \leq \theta c(\nu) 5^{\nu} \frac{e^{-\frac{r^{2}}{5 R^{2}}}}{V(x, R)} .
$$

According to [11, Lemma 3.10], the local doubling condition implies that for $r \geq$ $R$ :

$$
V(x, r) \leq\left(\theta 2^{\nu}\right)^{50+50 \frac{r}{R}} V(x, R)
$$

Hence we get that for some constant $\lambda_{1}$ depending only on $\theta, \nu, C$ :

$$
\int_{0}^{R^{2}} \int_{M \backslash B(x, R)} H(t, x, y) q(y) \operatorname{dvol}_{g}(y) d t \leq \lambda_{1} \int_{R}^{\infty} e^{-\frac{r^{2}}{5 R^{2}}} \frac{r}{V(x, r)} Q(x, r) d r .
$$

For the second part, we easily get by the same argumentation:

$$
\int_{0}^{r^{2}} \frac{e^{-\frac{r^{2}}{5 t}}}{t V(x, \sqrt{t})} d t \leq \theta \frac{r^{\nu}}{V(x, r)} \int_{0}^{r^{2}} \frac{e^{-\frac{r^{2}}{5 t}}}{t^{\frac{\nu}{2}+1}} d t \leq c(\nu) \theta \frac{1}{V(x, r)} .
$$

For the remaining part, we have to estimate:

$$
\begin{aligned}
\int_{0}^{R}\left(\int_{r^{2}}^{R^{2}} \frac{r e^{-\frac{r^{2}}{5 t}}}{t V(x, \sqrt{t})} d t\right) Q(x, r) d r d t & =\int_{0}^{R^{2}} \frac{1}{V(x, \sqrt{t})}\left(\int_{0}^{\sqrt{t}} \frac{r e^{-\frac{r^{2}}{5 t}}}{t} Q(x, r) d r\right) d t \\
& \leq \int_{0}^{R^{2}} \frac{Q(x, \sqrt{t})}{V(x, \sqrt{t})}\left(\int_{0}^{\sqrt{t}} \frac{r e^{-\frac{r^{2}}{5 t}}}{t} d r\right) d t \\
& \leq \int_{0}^{R^{2}} \frac{Q(x, \sqrt{t})}{V(x, \sqrt{t})}\left[-\frac{5}{2} e^{-\frac{r^{2}}{5 t}}\right]_{0}^{\sqrt{t}} d t \\
& \leq \frac{5}{2} \int_{0}^{R^{2}} \frac{Q(x, \sqrt{t})}{V(x, \sqrt{t})} d t=5 \int_{0}^{R} \frac{Q(x, r)}{V(x, r)} r d r
\end{aligned}
$$

Corollary 4.2. If $\left(M^{n}, g\right)$ is a closed Riemannian manifold with diameter $D$ and that satisfies the doubling $\left(D_{R}\right)$ and heat kernel estimate $\left(U E_{R}\right)$ at scale $R$ with $R \leq D$, then

$$
\kappa_{R^{2}}(q) \leq \lambda \sup _{x} \int_{0}^{D} r e^{-\frac{r^{2}}{7 R^{2}}}\left(f_{B(x, r)} q\right) d r .
$$


GEOMETRIC AND SPECTRAL ESTIMATES BASED ON SPECTRAL RICCI CURVATURE ASSUMPTIONS

Proof. We know that there is a positive constant $C$ such that if $\gamma=e^{C \frac{D}{R}}$ then

$$
\forall x \in M: \operatorname{vol}_{g}(M) \leq \gamma \operatorname{vol} B(x, D / 4) .
$$

Hence there is a finite set $A \subset M$ with $\# A \leq \gamma$ such that

$$
M=\cup_{a \in A} B(a, D / 2) .
$$

Hence for $x \in M$ and $r \geq D / 2$, we get:

$$
\int_{B(x, r)} q \leq \int_{M} q \leq \gamma \sup _{a \in A} \int_{B(x, D / 2)} q .
$$

And

Hence

$$
f_{B(x, r)} q \leq \gamma^{2} \sup _{a \in A} f_{B(x, D / 2)} q
$$

$$
\int_{D / 2}^{\infty} r e^{-\frac{r^{2}}{6 R^{2}}}\left(f_{B(x, r)} q\right) d r \leq \gamma^{2} 6 R^{2} e^{-\frac{D^{2}}{24 R^{2}}} \sup _{a \in A} f_{B(x, D / 2)} q .
$$

Similarly,

$$
\begin{aligned}
\int_{D / 2}^{D} r e^{-\frac{r^{2}}{7 R^{2}}}\left(f_{B(x, r)} q\right) d r & \geq \int_{D / 2}^{D} r e^{-\frac{r^{2}}{7 R^{2}}} d r \gamma^{-1}\left(f_{B(a, D / 2)} q\right) \\
& \geq 7 R^{2} e^{-\frac{D^{2}}{28 R^{2}} \gamma^{-1}}\left(f_{B(x, D / 2)} q\right)
\end{aligned}
$$

If we define

$$
\Gamma:=\sup _{x} \int_{0}^{D} r e^{-\frac{r^{2}}{7 R^{2}}}\left(f_{B(x, r)} q\right) d r
$$

we easily get for any $x \in M$ :

$$
\begin{aligned}
\int_{0}^{\infty} r e^{-\frac{r^{2}}{6 R^{2}}}\left(f_{B(x, r)} q\right) d r & \leq \Gamma+\gamma^{2} 6 R^{2} e^{-\frac{D^{2}}{24 R^{2}}} \sup _{a \in A} f_{B(a, D / 2)} q \\
& \leq \Gamma+\gamma^{3} e^{\frac{-D^{2}}{168 R^{2}} \Gamma .}
\end{aligned}
$$

There is a constant $c$ (depending only on $C$ ) such that if $R \leq D$ then

$$
\gamma^{3} e^{\frac{-D^{2}}{168 R^{2}}} \leq e^{\frac{-D^{2}}{168 R^{2}}+3 C \frac{D}{R}} \leq c
$$

Hence the result.

Theorem 4.3. There is a constant $\epsilon_{n}>0$ depending only on $n$ such that if $\left(M^{n}, g\right)$ is a closed Riemannian manifold such that some $R \leq D$ and such that for all $x \in M$ :

$$
\int_{0}^{D} r e^{\frac{-r^{2}}{7 R^{2}}}\left(f_{B(x, r)} \rho_{-}\right) d r \leq \epsilon_{n}
$$

then $(M, g)$ satisfies $\kappa_{R^{2}}\left(\rho_{-}\right) \leq \frac{1}{16 n}$. 
Proof. In [11], it has been shown that if $\kappa_{R^{2}}\left(\rho_{-}\right) \leq \frac{1}{16 n}$ then $\left(M^{n}, g\right)$ satisfies the condition $\left(\overline{\mathrm{D}_{R}}\right)$ and $\left(\overline{\mathrm{UE}_{R}}\right)$ with constants that depend only on $n:\left(\nu=e^{2} n\right)$.

i) $\forall x \in M, 0 \leq r \leq \rho \leq R: \frac{\operatorname{vol} B(x, \rho)}{\operatorname{vol} B(x, r)} \leq \theta_{n}\left(\frac{\rho}{r}\right)^{\nu}$.

ii) $\forall x, y \in M, t \in\left(0, R^{2}\right]: H(t, x, y) \leq \frac{C_{n}}{\operatorname{vol} B(x, \sqrt{t})} e^{-\frac{d^{2}(x, y)}{5 t}}$.

Hence there is a constant $\lambda_{n}$ depending only on $n$ such that

$$
\kappa_{R^{2}}\left(\rho_{-}\right) \leq \lambda_{n} \sup _{x \in M} \int_{0}^{D} r e^{\frac{-r^{2}}{7 R^{2}}}\left(f_{B(x, r)} \rho_{-}\right) d r .
$$

Hence if $R$ is the greatest real number such that $\kappa_{R^{2}}\left(\rho_{-}\right) \leq \frac{1}{16 n}$ then we get $\kappa_{R^{2}}\left(\rho_{-}\right)=\frac{1}{16 n}$ and necessarily

$$
\frac{1}{16 n} \leq \lambda_{n} \sup _{x \in M} \int_{0}^{D} r e^{\frac{-r^{2}}{7 R^{2}}}\left(f_{B(x, r)} \rho_{-}\right) d r .
$$

By contraposition, we get that if

$$
\sup _{x \in M} \int_{0}^{D} r e^{\frac{-r^{2}}{7 R^{2}}}\left(f_{B(x, r)} \rho_{-}\right) d r \leq \frac{1}{16 n \lambda_{n}}
$$

then

$$
\kappa_{R^{2}}\left(\rho_{-}\right) \leq \frac{1}{16 n}
$$

From the above we can obtain many results from [11] and [32], we prefer to refrain from stating all of them and concentrate on few of them:

Proposition 4.4. There is $a \eta_{n}$ such that if for all $x \in M$ :

$$
\int_{0}^{D} r\left(f_{B(x, r)} \rho_{-}\right) d r \leq \eta_{n}
$$

then the first Betti number of $M$ is smaller then $n$ :

$$
b_{1}(M) \leq n .
$$

Proposition 4.5. Assume that $\left(M^{n}, g\right)$ is a closed Riemannian manifold with diameter $D$ and introduce $\mathbf{I}(M, g)$ defined by

$$
\sup _{x \in M} \int_{0}^{D} r\left(f_{B(x, r)} \rho_{-}^{p}\right) d r=(\mathbf{I}(M, g) D)^{2(p-1)} .
$$

There is an explicit constant $\theta(n, p)$ such that for $R=\theta(n, p) D / \mathbf{I}(M, g)$ :

$$
\kappa_{R^{2}}\left(\rho_{-}\right) \leq \frac{1}{16 n}
$$

and with $\xi=\mathbf{I}(M, g) / \theta(n, p)$, one gets the isoperimetric inequality: for any open subset $\Omega \subset M$ with smooth boundary and $\operatorname{vol} \Omega \leq \frac{1}{2} \operatorname{vol} M$, we have

$$
1 \leq c_{n}^{1+\xi} D \operatorname{vol}(M) I_{n}(M)
$$


Proof. With the Hölder inequality, one easily gets (with $q=p /(p-1)$ ):

$$
\begin{aligned}
\int_{0}^{D} r e^{-\frac{r^{2}}{7 R^{2}}}\left(f_{B(x, r)} \rho_{-}\right) d r & \leq \int_{0}^{D} r e^{-\frac{r^{2}}{R^{2}}}\left(f_{B(x, r)} \rho_{-}^{p}\right)^{\frac{1}{p}} d r \\
& \leq\left(\int_{0}^{D} r e^{-q \frac{r^{2}}{7 R^{2}}} d r\right)^{\frac{1}{q}}(\mathbf{I}(M, g) D)^{\frac{2}{q}} \\
& \leq\left(\frac{7}{q} R^{2}\right)^{\frac{1}{q}}(\mathbf{I}(M, g) D)^{\frac{2}{q}} .
\end{aligned}
$$

The first result follows now easily from the Theorem 4.3 . The second result follows from Corollary 4.2 and Theorem 3.6. Indeed, we have

$$
\kappa_{R^{2}}\left(\rho_{-}^{p}\right) \leq \lambda_{n}(\mathbf{I}(M, g) D)^{2(p-1)} .
$$

\section{REFERENCES}

[1] M. Aizenman and B. Simon. Brownian motion and Harnack inequality for Schrödinger operators. Comm. Pure Appl. Math., 35(2):209-273, 1982.

[2] E. Aubry. Finiteness of $\pi_{1}$ and geometric inequalities in almost positive Ricci curvature. Ann. Sci. École Norm. Sup. (4), 40(4):675-695, 2007.

[3] Erwann Aubry. Finiteness of $\pi_{1}$ and geometric inequalities in almost positive Ricci curvature. Ann. Sci. École Norm. Sup. (4), 40(4):675-695, 2007.

[4] Erwann Aubry. Bounds on the volume entropy and simplicial volume in Ricci curvature $L^{p}$. bounded from below. Int. Math. Res. Not. IMRN, (10):1933-1946, 2009.

[5] D. Bakry and M. Ledoux. Sobolev inequalities and Myers's diameter theorem for an abstract Markov generator. Duke Math. J., 85(1):253-270, 1996.

[6] Dominique Bakry. L'hypercontractivité et son utilisation en théorie des semigroupes. In Lectures on probability theory (Saint-Flour, 1992), volume 1581 of Lecture Notes in Math., pages 1-114. Springer, Berlin, 1994.

[7] J. Barta. Sur la vibration fundamentale d'une membrane. C. R. Acad. Sci., 204:472-473, 1937.

[8] Vincent Bour and Gilles Carron. A sphere theorem for three dimensional manifolds with integral pinched curvature. Comm. Anal. Geom., 25(1):97-124, 2017.

[9] Peter Buser. A note on the isoperimetric constant. Ann. Sci. École Norm. Sup. (4), 15(2):213230, 1982.

[10] G. Carron. Inégalités de Hardy sur les variétés riemanniennes non-compactes. J. Math. Pures Appl. (9), 76(10):883-891, 1997.

[11] G. Carron. Geometric inequalities for manifolds with Ricci curvature in the Kato class. 2016. https://arxiv.org/abs/1612.03027 [math.DG].

[12] Jeff Cheeger. A lower bound for the smallest eigenvalue of the Laplacian. pages 195-199, 1970.

[13] Tobias Holck Colding. New monotonicity formulas for Ricci curvature and applications. I. Acta Math., 209(2):229-263, 2012.

[14] Xianzhe Dai, Peter Petersen, and Guofang Wei. Integral pinching theorems. Manuscripta Math., 101(2):143-152, 2000.

[15] K. D. Elworthy and S. Rosenberg. Manifolds with wells of negative curvature. Invent. Math., 103(3):471-495, 1991. With an appendix by Daniel Ruberman.

[16] Doris Fischer-Colbrie and Richard Schoen. The structure of complete stable minimal surfaces in 3-manifolds of nonnegative scalar curvature. Comm. Pure Appl. Math., 33(2):199-211, 1980. 
[17] S. Gallot. Inégalités isopérimétriques et analytiques sur les variétés riemanniennes. Astérisque, (163-164):5-6, 31-91, 281 (1989), 1988. On the geometry of differentiable manifolds (Rome, 1986).

[18] S. Gallot. Isoperimetric inequalities based on integral norms of Ricci curvature. Astérisque, (157-158):191-216, 1988. Colloque Paul Lévy sur les Processus Stochastiques (Palaiseau, 1987).

[19] David Gilbarg and Neil S Trudinger. Elliptic partial differential equations of second order. springer, 2015.

[20] B. Güneysu. Kato's inequality and form boundedness of Kato potentials on arbitrary Riemannian manifolds. Proc. Amer. Math. Soc., 142(4):1289-1300, 2014.

[21] T. Kato. Schrödinger operators with singular potentials. In Proceedings of the International Symposium on Partial Differential Equations and the Geometry of Normed Linear Spaces (Jerusalem, 1972), volume 13, pages 135-148 (1973), 1972.

[22] M. Ledoux. A simple analytic proof of an inequality by P. Buser. Proc. Amer. Math. Soc., 121(3):951-959, 1994.

[23] Ilaria Mondello. The local Yamabe constant of Einstein stratified spaces. Ann. Inst. H. Poincaré Anal. Non Linéaire, 34(1):249-275, 2017.

[24] William F. Moss and John Piepenbrink. Positive solutions of elliptic equations. Pacific J. Math., 75(1):219-226, 1978.

[25] P. Petersen, S. D. Shteingold, and G. Wei. Comparison geometry with integral curvature bounds. Geom. Funct. Anal., 7(6):1011-1030, 1997.

[26] P. Petersen and G. Wei. Relative volume comparison with integral curvature bounds. Geom. Funct. Anal., 7(6):1031-1045, 1997.

[27] Peter Petersen. On eigenvalue pinching in positive Ricci curvature. Invent. Math., 138(1):1-21, 1999.

[28] Peter Petersen and Chadwick Sprouse. Integral curvature bounds, distance estimates and applications. J. Differential Geom., 50(2):269-298, 1998.

[29] Peter Petersen and Guofang Wei. Analysis and geometry on manifolds with integral Ricci curvature bounds. II. Trans. Amer. Math. Soc., 353(2):457-478, 2001.

[30] Stefano Pigola, Marco Rigoli, and Alberto G. Setti. Vanishing and finiteness results in geometric analysis, volume 266 of Progress in Mathematics. Birkhäuser Verlag, Basel, 2008. A generalization of the Bochner technique.

[31] Christian Rose. Heat kernel estimates based on Ricci curvature integral bounds. Dissertation, Technische Universität Chemnitz, 2017.

[32] Christian Rose. Li-Yau gradient estimate for compact manifolds with negative part of Ricci curvature in the Kato class. 082016.

[33] Christian Rose and Peter Stollmann. The Kato class on compact manifolds with integral bounds on the negative part of Ricci curvature. Proc. Amer. Math. Soc., 145(5):2199-2210, 2017.

[34] Barry Simon. Schrödinger semigroups. Bull. Amer. Math. Soc. (N.S.), 7(3):447-526, 1982.

[35] Chadwick Sprouse. Integral curvature bounds and bounded diameter. Comm. Anal. Geom., 8(3):531-543, 2000.

[36] P. Stollmann and J. Voigt. Perturbation of Dirichlet forms by measures. Potential Anal., 5(2):109-138, 1996.

[37] J. Voigt. Absorption semigroups, their generators, and Schrödinger semigroups. J. Funct. Anal., 67(2):167-205, 1986.

[38] Jong-Gug Yun. A sphere theorem with integral curvature bounds. Kyushu J. Math., 56(2):225234, 2002.

E-mail address: Gilles.Carroneuniv-nantes.fr

E-mail address: christian.rose@mathematik.tu-chemnitz.de 